\title{
PROSTATE CANCER DETECTION AT REBIOPSY AFTER AN INITIAL BENIGN DIAGNOSIS: RESULTS USING SEXTANT EXTENDED PROSTATE BIOPSY
}

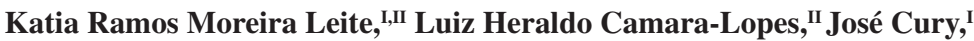 \\ Marcos F. Dall'Oglio, ${ }^{\mathrm{A}}$ Adriana Sañudo, ${ }^{\mathrm{I}}$ Miguel Srougi ${ }^{\mathrm{I}}$
}

doi: 10.1590/S1807-59322008000300009

Leite KRM, Camara-Lopes LH, Cury J, Dall'Oglio MF, Sañudo A, Srougi M. Prostate cancer detection at rebiopsy after an initial benign diagnosis: results using sextant extended prostate biopsy. Clinics. 2008;63:339-42.

INTRODUCTION: Sextant prostate biopsy remains the standard technique for the detection of prostate cancer. It is well known that after a diagnosis of small acinar proliferation (ASAP) or high grade prostate intraepithelial neoplasia (HGPIN), the possibility of finding cancer is approximately $40 \%$ and $30 \%$, respectively.

OBJECTIVE: We aim to analyze follow-up biopsies on patients who initially received a benign diagnosis after exclusion of HGPIN and ASAP.

METHODS: From July 2000 to December 2003, 1177 patients were submitted to sextant extended prostate biopsy in our hospital. The mean patient age was 65.5 years old, and the median number of fragments collected at biopsy was 13. HGPIN and ASAP were excluded from our study. We only considered patients who had a diagnosis of benign at the first biopsy and were subjected to rebiopsies up until May 2005 because of a maintained suspicion of cancer.

RESULTS: Cancer was initially detected in 524 patients (44.5\%), and the diagnosis was benign in 415 (35.3\%). Rebiopsy was indicated for 76 of the latter patients (18.3\%) because of a persistent suspicion of cancer. Eight cases of adenocarcinoma (10.5\%) were detected, six (75\%) at the first rebiopsy. Six patients were submitted to radical prostatectomy, and all tumors were considered clinically significant.

CONCLUSION: Our data indicate that in extended prostate biopsy, the first biopsy detects more cancer, and the first, second, and third rebiopsies after an initial benign diagnosis succeed in finding cancer in 7.9\% (6/55), 5.9\% (1/15) and 20\% (1/4) of patients, respectively.

KEYWORDS: Prostate. Cancer. Adenocarcinoma. Biopsy. Benign. Prostatic neoplasms.

\section{INTRODUCTION}

The systematic sextant prostate biopsy detects cancer in only $25-38 \%$ of patients ${ }^{1,2}$, and false-negative results have been reported to have an occurrence as high as $23 \%$. $^{3}$ Clinicians often face the dilemma of patients who present persistently abnormal levels of prostate-specific antigen (PSA)

'Laboratório de Investigação Médica da Disciplina de Urologia, LIM55, Faculdade de Medicina da Universidade de São Paulo - São Paulo/SP, Brazil. "Laboratório de Patologia Cirúrgica e Molecular, Hospital Sírio Libanês São Paulo/SP, Brazil.

katiaramos@uol.com.br

Received for publication on January 09, 2008

Accepted for publication on March 17, 2008 or an abnormal digital rectal examination, yet whose biopsies are persistently negative. When those patients are submitted to rebiopsy, cancer can be detected in $7-22 \%$ of cases at the first rebiopsy and $2-10 \%$ at the second rebiopsy. ${ }^{4-6}$

Two histological findings are predictive of prostatic adenocarcinoma: ASAP and HGPIN. ASAP is found in 5.5\% of prostate cancer cases, and can provide false positives due to marginal sampling or benign cell growth similar to an adenocarcinoma. A second biopsy detects cancer in up to $60 \%$ of patients. ${ }^{78} \mathrm{HGPIN}$ is the only pre-malignant lesion reported in $7 \%$ of needle biopsies, and rebiopsies detect cancer in $25-79 \%$ of cases after a diagnosis of HGPIN.

With the introduction of the extended biopsy, new rates of HGPIN, ASAP and cancer detection have been described. 
After a diagnosis of HGPIN, the possibility of diagnosing cancer in a rebiopsy dropped to an average of $21 \%$, while ASAP remains a strong predictor of cancer. ${ }^{9}$

Only one published study has analyzed detection rates for extended multi-site prostate biopsy. That study reported that rebiopsy, after an initial benign diagnosis, detected cancer in $13 \%$ of cases. ${ }^{5}$ Here we report cancer detection in rebiopsies after a first extended biopsy resulted in a benign diagnosis.

\section{MATERIALS AND METHODS}

From July 2000 to December 2003, 1682 biopsies were examined in our laboratory. Of these, 505 cases were sent for a second opinion and ultimately excluded from the study because of the relative prevalence of adenocarcinoma. The remaining 1177 biopsies were taken from men with PSA levels higher than $4 \mathrm{ng} / \mathrm{ml}$ or abnormal results from a digital rectal examination. Patients were 32-94 years old (mean, 65.5; median, 66). The number of biopsy cores ranged from nine to 32 (mean, 14; median, 13), and cores were taken bilaterally from the medial and lateral zones at the base, mid-gland, and apex of the prostate, as described by Presti et al. ${ }^{10}$

A benign diagnosis was given to 415 patients, and rebiopsies were performed on 76 of these patients because of persistent suspicion of cancer, increasing PSA levels, or abnormal digital rectal examinations. Rebiopsies were taken until May 2005. HGPIN- and ASAP-positive cases were excluded from the study, because our aim was to analyze the results of rebiopsy only in cases initially given a benign diagnosis.

The Mann-Whitney test was used to compare the PSA levels of patients whose rebiopsies revealed cancer with the levels of those with benign rebiopsies.

\section{RESULTS}

The results of the 1177 biopsies are shown in Table 1. Of all 1177 patients, 415 (35.3\%) received a benign diagnosis of prostatitis, normal prostatic tissue, atrophy, or glandular hyperplasia.

Table 2 - Rebiopsies after benign diagnosis in 76 patients
Table 1 - Results of 1177 biopsies examined between July 2000 and December 2003

\begin{tabular}{lcc}
\hline Diagnosis & $\mathrm{N}$ & $\%$ \\
\hline Adenocarcinoma & 524 & 44.5 \\
Benign & 415 & 35.3 \\
HGPIN & 181 & 15.4 \\
HGPIN + ASAP & 32 & 2.7 \\
ASAP & 25 & 2.1 \\
\hline
\end{tabular}

Among the 415 men receiving the benign diagnosis, mean age was 63.7 (median, 63; range, 49-82). The mean and median number of biopsy cores was 13 (range, 9 to 20).

PSA levels varied from 0.8 to $34 \mathrm{ng} / \mathrm{ml}$ (mean, $9.04 \mathrm{ng} /$ $\mathrm{ml}$; median, $8.00 \mathrm{ng} / \mathrm{ml})$. Of the 415 patients, 76 (18.3\%) were subjected to rebiopsies because of persistent suspicion of cancer. A total of 99 rebiopsies were performed. A single rebiopsy was performed in 53 patients $(69.7 \%)$, two were performed in 17 patients $(22.4 \%)$, three rebiopsies were performed in five patients $(6.6 \%)$, and four were conducted on a single patient (1.3\%).

The mean interval between the first and second biopsies was 20 months (median, 17; range, 1-49). The mean interval between second and third biopsies was 27 months (median, 23 ; range, 12-54), and the mean interval for the fourth biopsy was 36 months (median, 32; range, 22-57). One patient was submitted to a fifth biopsy 43 months after the first one. The results of rebiopsies are shown in Table 2.

Adenocarcinoma was detected in eight patients (10.5\%): six $(6 / 76)$ at the first rebiopsy, one (1/17) at the second rebiopsy, and one patient who was subjected to two rebiopsies and then to a transurethral resection (TUR) that led to a diagnosis of adenocarcinoma (1/5). The Gleason grade varied from 5 to 8 (mean, 6.75; median, 7). The mean percentage of tumor-positive fragments was $10.9 \%$ (median, 6.3\%; range, 5-18.8\%). The total percentage of tumor-positive fragments ranged from $1.3 \%$ to $6 \%$ (mean, $3.22 \%$; median, $3 \%$ ). The adenocarcinoma diagnosed in

\begin{tabular}{|c|c|c|c|c|c|}
\hline \multirow{2}{*}{ Rebiopsy } & \multicolumn{5}{|c|}{ Diagnosis } \\
\hline & Benign & Adenocarcinoma & PIN & ASAP & HGPIN+ASAP \\
\hline 1 & $55(72.4)$ & $6(7.9)$ & $11(14.5)$ & $3(3.9)$ & $1(1.3)$ \\
\hline 2 & $15(88.2)$ & $1(5.9)$ & $1(5.9)$ & - & - \\
\hline 3 & $4(80.0)$ & $1(20.0)^{*}$ & - & - & - \\
\hline 4 & $1(100.0)$ & - & - & - & - \\
\hline
\end{tabular}

*Adenocarcinoma diagnosed in TUR 
Table 3 - Results of radical prostatectomy of six patients

\begin{tabular}{lcccccccc}
\hline Patient & $\begin{array}{c}\text { PSA } \\
\mathrm{ng} / \mathrm{mL}\end{array}$ & $\begin{array}{c}\text { Weight } \\
(\mathrm{g})\end{array}$ & $\begin{array}{c}\text { Gleason } \\
\text { score }\end{array}$ & $\begin{array}{c}\text { Percentage } \\
\text { of tumor }\end{array}$ & $\begin{array}{c}\text { Volume } \\
(\mathrm{cc})\end{array}$ & $\begin{array}{c}\text { \% of Gleason } \\
\text { pattern 4 }\end{array}$ & $\begin{array}{c}\text { Tumor } \\
\text { location }\end{array}$ & $\begin{array}{c}\text { Stage } \\
\text { TNM 2002 }\end{array}$ \\
\hline 1 & 13.4 & 34.5 & $8(4+4)$ & 6 & 1.6 & 100 & PLBM & pT2bN0 \\
2 & 6.2 & 43.2 & $4(2+2)$ & 2 & 0.8 & 0 & TLM & pT2aN0 \\
3 & 13.1 & 98.9 & $5(3+2)$ & 1 & 0.9 & 0 & PLA & pT2aN0 \\
4 & 14.8 & 89.8 & $7(4+3)$ & 6 & 6.0 & 60 & PLM & pT2bN0 \\
5 & 9.2 & 102.9 & $7(4+3)$ & 4 & 4.2 & 64 & PRA & pT2aN0 \\
6 & 29.0 & 44.1 & $7(3+4)$ & 12 & 5.3 & 48 & PRLB & pT3aN0 \\
\hline
\end{tabular}

Note: In patients 1-4, adenocarcinoma was diagnosed at the first rebiopsy, in patient 5 at the second rebiopsy, and in patient 6 after TUR. PLBM, peripheral left base and medium; TLM, transitional - left medium; PLA, peripheral - left apex; PLM, peripheral - left medium; PRA, peripheral - right apex; PRLB, peripheral - right and left base

TUR had a Gleason score of $7(3+4)$ affecting $20 \%$ of the specimen (pT1b).

The PSA levels were not significantly different between patients who showed benign or malignant rebiopsy results. The mean and median PSA for patients with adenocarcinoma were, respectively, $11.9 \mathrm{ng} / \mathrm{ml}$ and $9 \mathrm{ng} / \mathrm{ml}$ (range, 6.15-34 $\mathrm{ng} / \mathrm{ml}$ ). For patients without carcinoma, the mean PSA was $8.9 \mathrm{ng} / \mathrm{ml}$ (median, 7.9; range, 0.8-24 ng/ml; $\mathrm{p}$ (with vs. without $)=0.452$ ).

Six patients were submitted to radical prostatectomy after a diagnosis of adenocarcinoma. All were considered clinically significant $(>0.5 \mathrm{cc}$, not confined, with Gleason patterns of at least 4 or 5). The results of radical prostatectomy are given in Table 3.

\section{DISCUSSION}

We have shown that, after extended sextant biopsies, $10.5 \%$ of patients who initially received benign diagnoses (but in whom cancer was a persistent suspicion) turned out to have detectable adenocarcinoma upon rebiopsy. Only the series published by Mian et al. contains data on extended biopsies, and they detected cancer in $13 \%$ of cases initially diagnosed as benign. ${ }^{5}$ The results published by Singh et al. ${ }^{7}$ were based on 12 core biopsies, and they reported a cancer detection rate of $21 \%$. In their study, however, they did not restrict their analysis to patients initially diagnosed as benign, and they also included HGPIN-positive cases, where HGPIN was the higher risk factor to find cancer in the rebiopsy.

The sensitivity and specificity of screening tests for prostate cancer cannot be determined with certainty because biopsies are not performed on patients with negative screening results. Nevertheless, it is known that as many as $19 \%$ of patients with initially negative prostate biopsies are found to have cancer upon rebiopsy.

It is interesting to note that, after a first extended biopsy, we diagnosed cancer in $44.5 \%$ of our patients, which is higher than the $25-38 \%$ classically reported for the sextant strategy. ${ }^{1,2}$ Studies based on the traditional strategy detect cancer in up to $30 \%$ of rebiopsies. ${ }^{11}$ These results show that diagnosis comes late for many patients, in addition to the fact that correct diagnosis requires repeated, invasive biopsies that are not without risk.

All data available in the literature are based on sextant biopsies, and the significance of basing results on 10 or more biopsy cores is not yet fully known. As suggested by O'Dowd et al., improving the success rate of biopsies may require increasing the number of fragments taken at the first prostate biopsy. ${ }^{12}$ Those authors argue that a better strategy for detecting cancer at the first biopsy is needed, given that they detected adenocarcinoma in almost $20 \%$ of their patients upon rebiopsy.

The post-sextant era has seen a change in the detection rate upon rebiopsy. A recent study by Moore et al. ${ }^{13}$ showed that when 10 or more cores are taken at the first biopsy, the possibility of finding cancer drops to $4.5 \%$ following a diagnosis of HGPIN. ${ }^{10}$ We published a similar study of rebiopsies in 53 patients positive for HGPIN, in which we reported cancer detection in $13.2 \%$ of the cases. ${ }^{6}$

Djavan et al. ${ }^{14}$ performed biopsies every six or eight weeks, and they detected cancer in $22 \%, 10 \%, 5 \%$ and $4 \%$ of four successive biopsies, respectively. While our study is closer to standard practice for urology outpatient care than theirs, their numbers are very similar to ours. The overall diagnosis of cancer in repeated biopsies was $7.5 \%$ in their study, compared to $10.5 \%$ in our case, almost all of them at the first rebiopsy. However, Djavan et al. suggested that, because of the difficulty of detecting extremely small tumors, biopsies beyond two were justified only for men at very high risk of developing cancer. Our data contradict this idea. All cancers we detected were clinically significant, including a patient in whom carcinonoma was diagnosed in a TUR following three biopsies. In this case the adenocarcinoma 
was pT3a, the Gleason score was $7(3+4)$, and tumor volume was $5.3 \mathrm{cc}$. Roehl et al. report similar results: by taking up to six biopsies, $99 \%$ of cancers were detected, and only $1 \%$ were considered clinically insignificant. ${ }^{15}$ Relative PSA levels can be informative in these situations, but the absolute levels are unhelpful for diagnosis. We have shown no significant difference in PSA between patients who, upon rebiopsy, were diagnosed with cancer and those who were not diagnosed with cancer. Increased PSA level does not always correspond to a diagnosis of cancer at rebiopsy. Mian et al. ${ }^{5}$ showed a positive biopsy rate of only $17 \%$ in the group that they considered to be at high risk of prostate cancer due to abnormal digital rectal examinations or a percentage of free PSA of $15 \%$ or less, or previous diagnosis of HGPIN or ASAP. The free-to-total prostate-specific antigen ratio may be diagnostically useful, although, according to Ciatto et al. ${ }^{16}$, this ratio should never be used to exclude rebiopsy in patients for whom suspicion of cancer persists.

The rebiopsies in our investigation were taken at longer intervals than in previous studies: the median was 17,23 , and 32 months for the first, second, and third rebiopsies, respectively. This is closer to the real practice of urologists, and it seems to be a reasonable scheme for a cancerscreening program. Morbidity associated with rebiopsies, particularly those conducted at short intervals, has been a concern in the past. However, postponing the biopsy procedure did not appear to pose a problem for our patients with cancer, almost all of whom had confined tumors. Roobol et al. came to the same conclusion. ${ }^{17}$ In a study of prostate rebiopsy of patients after an initial negative result, they detected cancer in $8 \%$ of cases after an interval of four years. The tumors in all cancer patients were confined to organs and were amenable to curative treatment.

In conclusion, we have shown that the sextant extended multisite prostate biopsy detects more cancer than the traditional sextant scheme. Of all the patients included in our study, $18 \%$ were rebiopsied after an initial benign diagnosis because of persistent suspicion of cancer, and malignancy was detected in $7.9 \%$ patients (6/55) at a second biopsy, $5.9 \%$ $(1 / 15)$ at a third biopsy, and $20.0 \%$ (1/4) at a fourth biopsy.

\section{REFERENCES}

1. Lui PD, Terris MK, McNeal JE, Stamey TA. Indications for ultrasoundguided transition zone biopsies in the detection of prostate cancer. $\mathrm{J}$ Urol. 1995;153:1000-3.

2. Terris MK. Extended field prostate biopsies: Too much of a good thing? Urology. 2000;55:457-60.

3. Murphy AM, McKiernan JM and Olsson CA. Controversies in prostate cancer screening. J Urol. 2004;172:1822-4.

4. Postma R, Roobol M, Schroder FH, van der Kwast TH. Lesions predictive for prostate cancer in a screened population: first and second screening round findings. Prostate. 2004;61:260-6.

5. Mian BM, Naya Y, Okihara K, Vakar-Lopez F, Troncoso P, Babaian RJ. Predictors of cancer in repeat extended multisite prostate biopsy in men with previous negative extended multisite biopsy. Urology. 2002;60:836-40.

6. Antunes AA, Freire GC, Aiello Filho D, Cury J, Srougi M. Analysis of the risk factors for incidental carcinoma of the prostate in patients with benign prostatic hyperplasia. Clinics. 2006;61:545-50.

7. Leite KR, Mitteldorf CA, Camara-Lopes LH. Repeat prostate biopsies following diagnoses of prostate intraepithelial neoplasia and atypical small gland proliferation. Int Braz J Urol. 2005;31:131-6.

8. Singh H, Canto EI, Shariat SF, Kadmon D, Miles BJ, Wheeler TM, et al. Predictors of prostate cancer after initial negative systematic 12 core biopsy. J Urol. 2004;171:1850-4.

9. Bostwick DG, Meiers I. Atypical small acinar proliferation in the prostate: clinical significance in 2006. Arch Pathol Lab Med. 2006 130:952-7.
10. Presti JC Jr. Prostate biopsy: how many cores are enough? Urol Oncol. 2003;21:135-40.

11. Borboroglu PG, Comer SW, Riffenbugh RH, Amling CL. Extensive repeat transrectal ultrasound guided prostate biopsy in patients with previous benign sextant biopsies. J Urol. 2000;163:158-62.

12. O’Dowd GJ, Miller MC, Orozco R, Veltri RW. Analysis of repeated biopsy results within 1 year after a noncancer diagnosis. Urology. 2000;55:553-9.

13. Moore CK, Karikehalli S, Nazeer T, Fisher HAG, Kaufman RP, Mian BM. Prognostic significance of high grade prostatic intraepithelial neoplasia and atypical small acinar proliferation in the contemporary era. J Urol. 2005;173:70-2.

14. Djavan B, Ravery V, Zlotta A, Dobronski P, Dobrovits M, Fakhari M. et al. Prospective evaluation of prostate cancer detected on biopsies 1, 2, 3 and 4: When should we stop. J Urol. 2001;166:1679-83.

15. Roehl KA, Antenor JA, Catalona WJ. Serial Biopsy results in prostate cancer screening study. J Urol. 2002;167:2435-9.

16. Ciatto S, Lombardi C, Rubeca T, Zappa M. Predictors of random sextant biopsy outcome in screened men with PSA $>4 \mathrm{ng} / \mathrm{mL}$ and negative sextant biopsy at previous screening. Experience in a population-based screening program in Florence. Int J Biol Markers. 2004;19:89-92.

17. Roobol MJ, van der Cruijsen IW, Schroder FH. No reason for immediate repeat sextant biopsy after negative initial sextant biopsy in men with PSA level of $4.0 \mathrm{ng} / \mathrm{mL}$ or greater. Urology. 2004;63:892-7. 